Berliner Poliklinik ergab bereits damals eine totale linksseitige Recurrenslähmung. Da sofort der Verdacht auf ein Aortenaneurysma auftauchte, wurde der Kranke mit Röntgenstrahlen durchleuchtet, wobei sich ein pulsirender Tumor in der Gegend des Manubrium sterni fand. Auf diesen Befund hin wurde die sichere Diagnose „A or tenane urysma" gestellt. Die Recurrenslähmung besserte sich nicht, seit dem Januar dieses Jahres stellten sich aber anderweitige Beschwerden ein, Schmerzen in den oberen Partieen von Brust und Rücken und Beschwerden beim Schlucken, und zwar besonders beim Genuss fester Speisen. Erbrechen trat nicht auf. Die geschilderten Beschwerden nahmen allmählich an Intensität zu, die Ernährung wurde immer schwieriger, und das Körpergewicht ging schnell zurück (von 157 auf 130 Pfund). Patient wurde daher unter der Diagnose eines den Oesophagus verengernden Aneurysmas der Aorta dem Krankenhaus überwiesen. Von ätiologischen Momenten liessen sich weder Branntweingenuss, noch Lues, noch ein Trauma feststellen

Status praesens: Mässig fiebernder, stark abgemagerter Mann von blasser, fahler Hautfarbe. Knochenbau gracil, Muskulatur schwach, Fettpolster fehlt. Haar schwarz, graumelirt. Puls nicht beschleunigt, kräftig, regelmässig, es besteht leichte Arteriosklerose. Athmung von normaler Frequenz, bei erhöhter Rückenlage ohne Beschwerden und hinreichend ausgiebig, beiderseits gleich, bei horizontaler Rückenlage tritt leichte Athemnoth ohne Stridor ein. Keine Exantheme, keine Oedeme, keine Venenstauungen. Am Halse, in den Achselhöhlen, den Ellenbogen- und den Schenkelbeugen finden sich perlschnurartig angeordnete, nicht schmerzhafte ge schwoll en e Drüsen von Linsen-bis Erbsengrösse.

Kopf: Pupillen beiderseits gleich, mittelweit, reagiren. Con junctiven anämisch. Brechende Medien klar. Papillen scharf umrandet, prominent, so dass die Gefässe im Bogen über den Rand hinwegziehen, von normaler Farbe. Retina ohne Veränderungen. Augenbewegungen völlig frei. Gesichtsnerven intact. Rachenorgane normal.

Kehl ko pf: Schleimhaut von blasser Farbe, beide Stimmbänder glatt,

IV. Aus der inneren Abtheilung des städtischen Krankenhauses in Charlottenburg.

(Dirigirender Arzt: Prof. Dr. E. Grawitz.)

\section{Ist die Durchleuchtung mit Röntgenstrahlen ausschlaggebend für die Differentialdiagnose zwischen Aortenaneurysma und intra- thoracischem Tumor?}

\section{Von Dr. E. Gebauer, Assistenzarzt.}

Bei der Entdeckung der Röntgenstrahlen war es vorauszusehen, dass diese neue Bereicherung der Naturwissenschaft in erster Linie unter den Medicinern dem Chirurgen praktisch zu gute kommen würde. Entgegen manchem zu Anfang geäusserten Zweifel hat die rapide Entwickelung der Technik auf diesem Gebiet aber schon jetzt den Erfolg gehabt, dass nunmehr auch der innere Mediciner die Durchleuchtung des Körpers mit Röntgenstrahlen als ein nicht unwichtiges diagnostisches Hilfsmittel ansehen kann, wenn es sich um die Diagnose von Tumoren, um die Bestimmung der Grenzen compacter Organe, um den Nachweis von Verdichtungen im Lungengewebe $u$. a. handelt. Insbesondere bei dem Aortenaneurysma schien die neue Methode geeignet, durch den direkten Nachweis der pulsirenden Geschwulst die Sicherung dieser Diagnose gegenüber der Annahme intrathoracischer Tumoren zu ermöglichen. Und in der That kann oft genug das Röntgenbild den Beweis liefern für die Richtigkeit einer auf Grund anderer klinischer Symptome gestellten Wahrscheinlichkeitsdiagnose auf Aortenaneurysma. Wie aber, wenn der Befund der Röntgendurchstrahlung im Widerspruch steht mit den Resultaten der Percussion, Auscultation, Palpation etc.? Ist man da berechtigt, unter Vernachlässigung der gesicherten Ergebnisse dieser altbewährten Methoden die Röntgendurchleuchtung einseitig zubevorzugen und sie als ausschlaggebenden Factor bei der Differentialdiagnose anzusprechen?

Einen Beitrag zur Entscheidung dieser Frage möge folgender im städtischen Krankenhause zu Charlottenburg beobachtete Fall bilden.

Am 27. Februar h. a. wurde der Wärter Friedrich G. in das hiesige Krankenhaus aufgenommen.

Anamnese: Erbliche Belastung und frühere Krankheiten fehlen. Die jetzige Krankheit begann im September vorigen Jahres (also vor etwa fünf Monaten) damit, dass der Kranke eines Tages, wie er sagt, ganz plötzlich, während des Frühstückessens, völlig heiser wurde. Weitere Beschwerden will er damals nicht gehabt haben, eine Ursache der Erkrankung weiss er nicht anzugeben. Die Untersuchung in einer elfenbeinweiss, das rechte frei beweglich. Das linke Stimmband steht unbeweglich in Cadaverstellung und bewegt sich weder beim Athmen noch beim Anlauten. Die Sprache ist völlig heiser, aber verständlich. Palpirt man den Kehlkopf während des Sprechens, so fühlt man die Resonanz der rechten Kehlkopfhälfte stärker als die der linken. Drängt man den Kehlkopf nach oben, so fühlt man deutlich, wie er, der Herzsystole entsprechend, rhythmisch nach unten gezogen wird (Oliver'sches Symptom).

Thorax: Der Thorax ist lang und schmal gebaut, die Intercostalräume weit, die Gruben tief. In beiden Oberschlüsselbeingruben linsengrosse, harte Lymphknoten. Beide Thoraxhälften sind gleich gebaut und dehnen sich bei der Athmung gleichmässig aus.

Lungen: Die obere Lungengrenze steht rechts - vorn wie hinten - $1 \mathrm{~cm}$ tiefer als links, der Percussionsschall ist über der rechten Oberschlüsselbeingrube kürzer als links. Die unteren Lungengrenzen stehen beiderseits gleich hoch und sind gleichmässig bei der Athmung verschieblich. Die Athmung ist ïberall vesiculär, über der rechten Spitze leicht verschärft, in den abhängigen Partieen der rechten Lunge begleitet von vereinzelten leisen pleuritischen Schabegeräuschen, sonst iiberall ohne Nebengeräusche.

Herz und Gefässsystem: Die Herzdämpfung reicht nach rechts bis zum linken Brustbeinrand, nach links bleibt sie $1 / 2 \mathrm{~cm}$ innerhalb der Mamillarlinie, nach oben wird sie vom Oberrand der vierten Rippe begrenzt. Ferner findet sich über dem Manubrium sterni eine etwa fünfmarkstückgrosse Stelle mit leichter Schallabschwächung ohne sichtbare oder fühlbare Pulsation und ohne Schwirren. Zwischen den Schulterblättern besteht keine Schallabschwächung. Der Schwertfortsatz ist trichterförmig nach innen eingesunken. Die Herztöne sind an der Mitralis und im dritten linken Zwischenrippenraum begleitet von leisen systolischen Geräuschen, an den übrigen Ostien sowie im Gebiet der Dämpfung über dem Manubrium sterni sind die Töne völlig rein. Der Puls ist an beiden Subclavien, beiden Carotiden, beiden Radialarterien und beiden Cruralarterien synchron und beiderseits gleich stark.

Beim Schlucken klagt Patient über mässige Schmerzen, doch gelingt es ihm ohne Schwierigkeit, flüssige und weiche Nahrungsmittel herunterzubringen. Von einer Sondirung der Speiseröhre wurde mit Rücksicht auf die Möglichkeit eines Aortenaneurysmas Abstand genommen.

Abdomen: Bauchdecken weich, keine Resistenz, keine Dämpfung, keine Druckempfindlichkeit, kein Ascites. Die Leberdämpfung überragt den Rippenbogen um $1 / 2$ Fingerbreite, die Leber ist hart, aber nicht höckrig und nicht schmerzhaft. Milz nicht vergrössert. Rechtsseitige Leistenbruchanlage. Genital ohne Veränderungen, keine Zeichen alter Lues.

Extremitäten ohne Veränderungen.

Reflexe erhalten (Patellarreflexe, Plantarreflexe, Cremasterreflexe). Keine Störungen der Sensibilität oder Motilität. Durch Beklopfen der Muskeln werden auffällig starke fibrilläre Muskelzuckungen ausgelöst.

Urin frei von Eiweiss und Zucker, Indicanreaction negativ, bei 
der Diazoreaction entsteht zunächst ein schwach röthlicher Schüttelschaum, der bei längerem Stehen völlig weiss wird.

Stuhlgang ohne Besonderheiten.

Auswurf spärlich, schleimig, ohne Blutbeimengungen, enthält weder elastische Fasern, noch Tumorzellen, noch Tuberkelbacillen.

Im Blut findet sich eine leichte Vermehrung der weissen Blutzellen, keine kernhaltigen oder gekörnten rothen Blutkörperchen.

Bei diesem Befunde konnte die Differentialdiagnose schwanken zwischen einem intrathoracischen Tumor, einer Lungenphthise mit starker Drüseninfiltration und einem Aortenaneurysma.

Für Tumor sprach: die Dämpfung über dem Sternum, die Cachexie, die Lymphdrüsenschwellungen, die Dysphagie, das Fehlen manifester Tuberkuloseerscheinungen und das Fehlen der für Aortenaneurysma charakteristischen Pulsveränderungen, dagegen: das Fieber und das Fehlen von Venenstauungen,

für Lungenphthise: der Befund auf der rechten Lungenspitze, das Fieber, die Cachexie und die Drüsenschwellungen, dagegen: das Fehlen von Tuberkelbacillen und schwereren Lungenerscheinungen und das Oliver'sche Symptom,

für Aortenaneurysma: die Dämpfung auf dem Sternum und die Pulsation des Kehlkopfes, dagegen: vor allem die Cachexie, die sich allerdings durch mangelhafte Ernährung in Folge mechanischerVerengerungdes

Oesophagus durch den Druck eines Aneurysmas deuten liess, ferner die Drüsenschwellungen, die man durch eine alte Lungentuberkulose erklären konnte, schliesslich das Fehlen aller örtlichen und peripheren Erscheinungen eines Aneurysmas (Schwirren, systolische oder diastolische Geräusche und Pulsabnormitäten), ferner das Fieber.

Die Recurrenslähmu ng liess alle drei Diagnosen $\mathrm{zu}$, wenn auch die Thatsache, dass von vorn herein nicht nur die Abductoren, sondern die sämmtlichen linksseitigen Kehlkopfmuskeln gelähmt waren, viel mehr für einen Tumor als für ein Aneurysma sprach. Das Oliversche Symptom, das

früher als entscheidend für die Diagnose „Aneurysma des Arcus aortae“ galt, konnte, wie Fraenkel1) und nach ihm Auerbach ${ }^{2}$ ) gezeigt haben, sowohl für Aneurysma als für Tumor, bezw. eine grössere infiltrirte Lymphdrüse sprechen.

Am wenigsten Wahrscheinlichkeit hatte die Annahmo einer einfachen Phthise mit stark vergrösserten Bronchialdrüsen, $n$ ach den klinischen Erscheinungen sprach alles für eine maligne Neubildung in der Thoraxhöhle neben einer alten abgeheilten Phthise, doch fiel dagegen die durch die Anamnese gewonnene Kenntniss von der Anwesenheit eines pulsirenden Tumors im Thorax stark ins Gewicht. So wurde denn, nachdem der Kranke zunächst durch Bettruhe und zweckentsprechende Ernährung unter Joddarreichung gebessert war, am 1. März eine Durchleuchtung mit Röntgenstrahlen vorgenommen.

Auf den Reproductionen des dabei gewonnenen Bildes, von denen Fig. 85 die Ansicht von vorn, Fig. 86 die Ansicht von hinten darstellt, lässt sich der Schatten eines Tumors erkennen, dessen Grenze vom linken Herzen aus etwa in der Mitte zwischen linkem Sternalrand und Knorpelgrenze der Rippen fast geradlinig in die Höhe steigt und am Unterrand der zweiten Rippe in nach aussen convexem Bogen medianwärts umbiegt. Rechts sind die Contouren die entsprechend nach unten zurück verlaufen, nicht so scharf

1) Deutsche medicinische Wochenschrift 1899, No. 1.

2) Deutsche medicinische Wochenschrift 1900 , No. 8 ausgeprägt wie links. Dieser dem Herzen direkt aufsitzende Tumor zeigte auf dem Schirm deutliche Pulsation. Dieser Befund schien so charakteristisch, dass auch jetzt wieder von einer auf dem Gebiete der Skiagraphie autoritativen Seite die Diagnose auf Aortenaneurysma mit Sicherheit gestellt wurde.

Trotzdem und obwohl eine in der ersten Behandlungswoche erzielte Gewichtszunahme um $0,7 \mathrm{~kg}$ ebenfalls gegen eine maligne Neubildung zu sprechen schien, blieb die klinische Wahrscheinlichkeitsdiagnose „intrathoracischer Tumor" bestehen, wenn auch für den pulsirenden Tumor des Röntgenbildes keine sichere Erklärung gefunden wurde.

Nach ganz vorübergehender Besserung verschlimmerte sich der Zustand des Kranken bald wieder, Patient verfiel mehr und mehr, es stellte sich stärkere Dyspnoë ein, und etwa vier Tage vor dem Tode bildete sich ein rechtsseitiges pleuritisches Exsudat, das am Tage vor dem Tode (12. März) stark anwuchs. Die Probepunction ergab ein seröses, eitrig getrübtes Exsudat, das in dem reichlichen Sediment massenhaft Leukocyten und zahlreiche runde und ovale, theilweise abgeplattete Epithelzellen ohne eine für Carcinom charakteristische Anordnung enthielt. Die bacteriologische Untersuchung ergab sehr spärliche Pneumo-

Fig. 86.

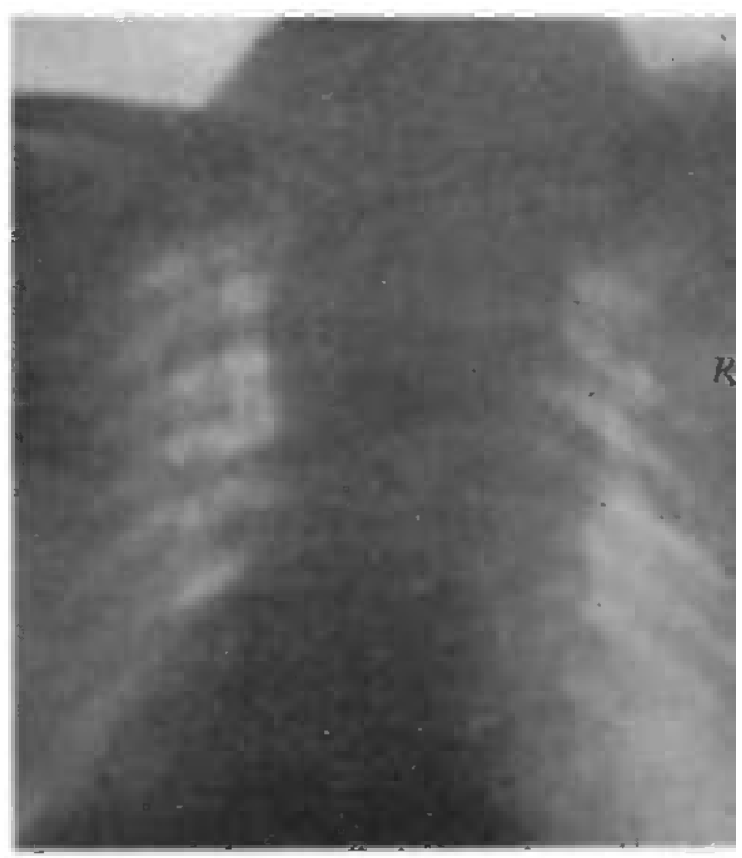

coccen, dagegen weder Tuberkelbacillen noch Strepto- oder Staphylococcen. Unter den Erscheinungen zunehmender Herzschwäche und Dyspnoë führte der Zustand am 13. März zum Tode. In der Agone traten noch rechtsseitige geringe Stauungserscheinungen im Bezirk der thoracalen Hautvenen auf.

Die Section wurde 36 Stunden post mortem vorgenommen. Aus dem Protokoll lasse ich die wesentlichen Punkte folgen.

Bei Eröffnung der Brusthöhle findet sich, dass die rechte Pleura pulmonalis durch zahlreiche frische Adhäsionen und einzelne alte Stränge mit der Pleura costalis verwachsen ist; in der rechten Pleurahöhle finden sich etwa 1400-1500 ccm einer serösen, stark eitrig getrübten und Fäden enthaltenden Flüssigkeit von fötidem Geruch. Die linke Lunge ist im Unterlappen durch einige leicht lösliche frische Verklebungen an die Pleura costalis angelöthet, im linken Pleurasack findet sich keine freie Flüssigkeit.

Hals- und Brustorgane werden zusammen herausgenommen, was ohne Schwierigkeit gelingt. Dabei finden sich grosse peritracheale und peribronchiale Drïsenpackete.

Die Schleimhaut des Pharynx und des Kehlkopfes ist blass: zeigt sonst keine Veränderungen, ebensowenig die Schilddrüse.

In der Speiseröhre findet sich $9 \mathrm{~cm}$ unterhalb der Plica interarytaenoidea ein kreisrunder Krater von $4,5 \mathrm{~cm}$ Durchmesser. der nach links in eine überall geschlossene, etwa wallnuss. grosse Höhle führt. Die Ränder des Kraters sind wallartig erhaben, überall ulcerirt, die Wände der Höhle dagegen glatt. $3 \mathrm{~cm}$ unter dem' Unterrande dieses Kraters findet 
sich ein zweiter, grösserer, ebenfalls kreisrunder Krater von $7 \mathrm{~cm}$ Durchmesser, der ebenfalls überall wallartig pro minirende und ulcerirte Ränder hat und die Oeffnung einer nach rechts sich erstreckenden apfelgrossen Höhle bildet. die mit jauchigriechenden, zerfallenen Massen und Gewebs fetzen angefüllt ist. Unter dem Wasserstrahl lassen diese nekrotischen Massen sich leicht abspülen, und die überall zerfressenen, ausgebuchteten Wände der Höhle werden frei. Der angrenzende Mittellappen der rechten Lunge ist durch feste pleuritische Adhäsionen mit dem Tumor verwachsen, und an einer Stelle findet sich eine Perforation des zerfallenen Tumors in das Lungengewebe, das dadurch in mehr als apfelgrosser Ausdehnung in eine weiche, fötidriechende, graugrüne, gangränöse Masse verwandelt ist. Die unteren Partieen des Oesophagus sind normal, die Schleimhaut zeigt nur in der unmittelbaren Umgebung der Geschwürskrater Injection. Oberhalb des unteren Kraters ist der Oesophagus erweitert. Die Maasse betragen dicht unter dem Kehlkopf . . . . . 4,5 cm am oberen Rande des oberen Kraters 6 in der Mitte des oberen Kraters . . 8,2 $", \quad$ zwischen beiden Kratern 11 am Unterrand des unteren Kraters . . 14 $5 \mathrm{~cm}$ unter dem unteren Krater $: 7$

\section{Fig. 87.}

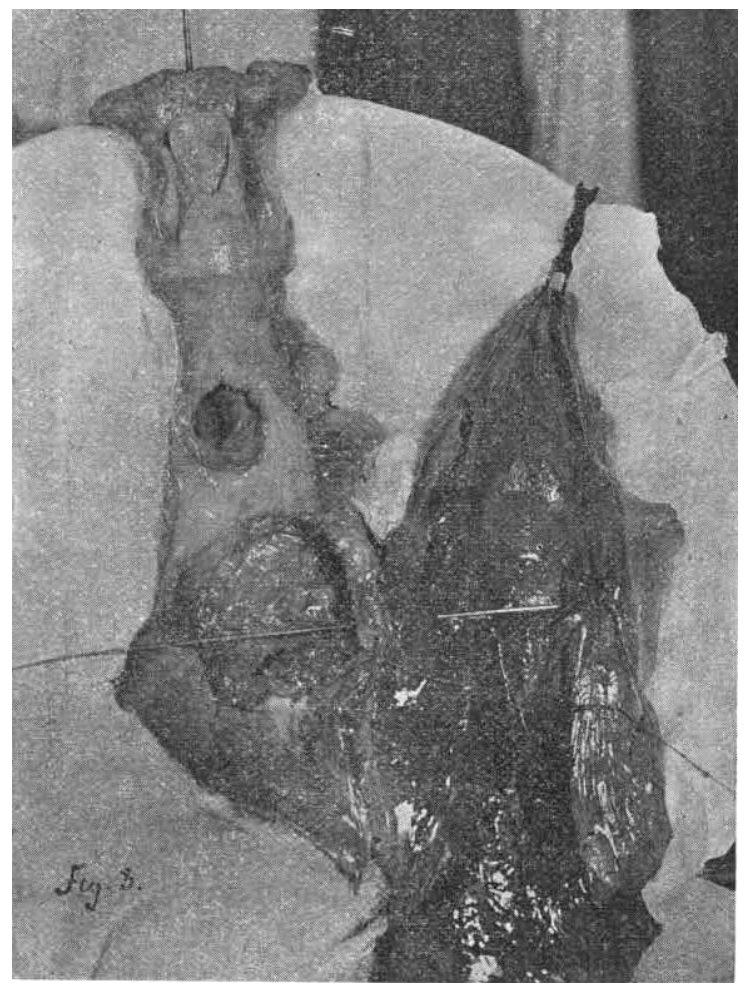

Ansicht der aufgeschnittenen Speiseröhre und der rechten Lunge von hinten. Die angrenzenden Organe sind bis auf eine in der Mitte aufoeschnittene Trachealdrüse, die in dem Winkel zwischen Speiseröhre und Lunge sichtbar ist, durch Gaze verdeckt. Man sieht die beiden Tumorenkrater, die von dem unteren Tumor in die Lunge eingeführte Sonde markirt die Perforationsstelle und die Stelle der Lungengangrän. Die weissen Flecke sind Lichtreflexe.

Beide Lungen, besonders die rechte, sind stark ödematös und sehr blutreich. Im Oberlappen der linken Lunge findet sich eine etwa haselnussgrosse narbig eingezogene Stelle, die auf dem Durchschnitt schiefrige Induration mit mehreren stecknadelknopfgrossen Kalkeinlagerungen aufweist. Der rechte Oberlappen ist stark geschrumpft, schiefrig indurirt, ohne Erweichungsheerde. Im Mittel- und Unterlappen finden sich einzelne bohnen- bis haselnussgrosse, scharf abgegrenzte, auf dem Durchschnitt röthlichweisse, zum Theil fettig zerfallene runde Knoten. Die Umgebung der oben geschilderten gangränösen Partie lässt eine Zone frischer rother Hepatisation erkennen.

Im Herzbeutel finden sich etwa $150 \mathrm{ecm}$ einer serösen, eitrig getrübten, fadenhaltigen Flüssigkeit, und auf dem Epicard und an der Innenfläche des Pericards massenhafte fibrinös-eitrige Niederschläge. Endocard und Klappen überall intact; der Herzmuskel von normaler Grösse, mässig straff contrahirt, in geringem Grade braun atrophisch. Die Aorten- und Pulmonalklappen ebenfalls intact. Die Aorta ist am Arcus durch ein zwischen ihr und dem oberen Oesophagustumor liegendes Drüsenpacket in mässigem Grade von oben nach unten comprimirt, die Aorta ascendens nur sehr wenig dilatirt, die Aorta descendens von normaler Weite. Die Aortenwandung weist an einzelnen Stellen, besonders an der comprimirten Partie, einzelne linsen- bis erbsengrosse, theils verfettete, theils verkalkte Heerde auf. Die Maasse der Aorta betragen:

dicht über den Semilunarklappen $9,5 \mathrm{~cm}$ in der Mitte zwischen Semilunarklappen und Trunc. anonym. . 10 am Aboang des Truncus anonymus am Arcus aortae (Compressionsstelle) 7,7 in der Mitte zwischen Carotis sinistra und Subclavia sinistra . 7,8 Aorta descendens an verschiedenen Stellen Nirgends findet sich die Andeutung eines Aneurysmas.

Die peritrachealen Lymphdrüsen sind beiderseits mehr oder weniger stark geschwollen, auffallend gross ist ein Packet, das zwischen dem linken Schilddrüsenlappen und dem oben geschilderten oberen Tumordivertikel des Oesophagus liegt, ferner eine rechts neben der Trachea etwa $5 \mathrm{~cm}$ unter der Schilddrüse liegende, über wallnussgrosse, infiltrirte und central erweichte Drüse und Gruppen von kleineren Drüsen, die zwischen dieser und dem unteren Speiseröhrentumor liegen.

Was die gegenseitigen Beriehungen der geschilderten Organe anlangt, so ist von Wichtigkeit, dass die laterale Wand des oberen Speiseröhrendivertikels (bezw. Tumors) mit der Hinterwand des Arcus aortae durch ein Drüsenpacket fest verwachsen ist, ferner ist die vordere Wand der Speiseröhre an der Mitte des unteren Tumors mit der Aolta descendens verlöthet, und schliesslich ist der unterste Abschnitt der Trachea und der obere Theil des linken Bronchus an seiner linken Seite fest mit der hinteren und unteren Partie des Arcus aortae verbunden. Auch diese Fixation wird bedingt durch ein Drüsenpacket, das aus bohnen-bis haselnuss grossen, fest infiltrirten Drüsen besteht und nach rechts und links mit den beiden genannten Organen, nach oben mit der unteren Wand des mehrfach erwähnten oberen Speiseröhrentumordivertikels verwachsen ist. In dieses Drüsenpacket ist der linke Nervus reculrens fest eingemauert. Fine Compression der Trachea oder des Bronchus besteht nicht.

Vom Befunde der Bauch- und Beckenorgane sei hier nur kurz erwähnt, dass die retroperitonealen und mesenterialen Drüsen ebenfalls in verschiedener Ausdehnung infiltrirt waren, dass aber Milz, Nieren, Leber, Magendarmtractus und Genital frei von Krebserscheinungen waren. Ferner bestand eine frische parenchymatöse Nephritis

Die mikroskopische Untersuchung der ulcerirten Oesophagustunoren, der infiltrirten Drüsen und der Lungenmetastasen ergab überall einen rein epithelialen Bau der Tumoren, stellenweise mit ausgeprägt zwiebelartiger Anordıung der Zellen.

Die Entwickelung der Krankheit war somit also folgendermaassen vor sich gegangen: Das primär entstandene untere Oesophaguscarcinom hatte die regionären Lymphdrüsen infiltrirt, hatte Metastasen in der Lunge und eine niehr nach oben gelegene Metastase in der Speiseröhre gesetzt. Die zwischen Alcus aortae und linkem Bronchus gelegenen Lymphdrüsen hatten den linken Recurrens geschädigt und damit das erste Symptom, die Kehlkopferscheinungen hervorgerufen, sie hatten bei fortschreitendem Wachsthum die Fixation des linken Bronchus an den Arcus a ortae herbeigeführt und so das Oliver'sche Symptom bedingt; der weiter um sich greifende Plimärtumor hatte zunächst pleuritische Adhäsionen mit der rechten Lunge verursacht, war dann in den Mittellappen perforirt und hatte hier zu Gangrän und reaktiver Entzündung der Umgebung geführt. Durch eine Perforation der Pleura war schliesslich sub finem das jauchige Exsudat entstanden, an dem der Kranke gestorben ist.

So weit lassen sich der Prozess und seine Erscheinungen völlig zwanglos und leicht erklären. Schwieriger ist die Deutung des auf den Röntgenbildern sichtbaren pulsirenden Tumors. Die auf den oben wiedergegebenen Röntgenphotographieen sichtbaren dunklen Tumorschatten finden ihre Erklärung ja auch in der Summe der beiden grossen Oesophagustumoren mit den zahlreichen neben- und übereinander liegenden kleineren oder grösseren infiltrirten Lymphknoten. Für das Phänomen der Pulsation dieses Tumorschattens bleibt mir nur die Annahme, dass der auf der oberen "Wand des Arcus aortae fest aufsitzende kleinere obere Oesophagustumor bei der Systole von der Aorta nach oben geworfen und der grössere, der hinteren und seitlichen Wand der Aorta descendens angeheftete untere Speiseröhrenkrebs bei der Systole nach rechts verschoben wurde. Diese pulsatorische Verschiebung, die in Wirklichkeit nur eine minimale sein konnte, muss bei der Projection auf den Schirm die Pulsation eines Aortenaneurysmas vorgetäuscht haben.

Es liegt mir natürlich fern, den unzweifelhaft grossen Werth, 
den die Durchleuchtung des Thorax bei einem Verdacht auf Aortenaneurysma haben kann, irgendwie in Abrede zu stellen, doch glaube ich an der Iland eines Falles wie des vorliegenden, Bedenken dagegen äussern zu müssen, dass man gegenüber dem Fehlen anderer wichtiger klinischer Erscheinungen allein auf den Befund der Röntgendurchstrahlung die Diagnose aufbauen zu können glaubt. Es bleibt abzuwarten, wie eine weitere Vervollkommnung der skiagraphischen Technik die Sicherheit der Diagnose zu erhöhen im Stande sein wird, inwieweit besonders die Durchleuchtung des Thorax in schräger Richtung (von hinten links nach vorn rechts), wie sie neuerdings Holzknecht') in der Nothnagel'schen Klinik angewendet, sich als brauchbar erweisen wird, zunächst ist jedenfalls der Ausfall der Durchleuchtung des Thorax mit Röntgenstrahlen nicht als allein ausschlaggebend für die Differentialdiagnose $z$ wischen Aortenaneurysma und Mediastinaltumor anzuerkennen.

Zum Schluss gestatte ich mir, Herrn Prof. Dr. Grawitz für seine Anregung und die gütige Ueberlassung des Materials meinen gehorsamen Dank auszusprechen. 